\title{
To Compute the Optimal Interpolation Formula
}

\author{
By P. W. Gaffney
}

\begin{abstract}
The purpose of this paper is to explain how to compute the function $\Omega$ which interpolates values of a function of one variable $f(x)$ at $n$ distinct points $x_{1}$ $<x_{2}<\cdots<x_{n-1}<x_{n}$, and which, whenever $\| f^{(k)_{\|_{\infty}}}$ is bounded and the value of the bound is unknown, provides the smallest possible value of $B(x)$ in the error bound
\end{abstract}

$$
|f(x)-\Omega(x)| \leqslant B(x)\left\|f^{(k)}\right\|_{\infty} .
$$

1. Introduction. Gaffney (1976b) and Gaffney and Powell (1975) considered the solution to the following problem.

Given values of a function of one variable, $f(x)$, at $n$ distinct points: $x_{1}<x_{2}$ $<\cdots<x_{n-1}<x_{n}$ and given that $\left\|f^{(k)}\right\|_{\infty}, 1 \leqslant k \leqslant n$, is bounded, but that the value of the bound is unknown, identify the interpolating function $\Omega$ and the function $B$, which satisfies the inequality

$$
|f(x)-\Omega(x)| \leqslant B(x)\left\|f^{(k)}\right\|_{\infty},
$$

where for all $x$ in the range $x_{1} \leqslant x \leqslant x_{n}, B(x)$ is as small as possible.

They solved this problem and they called the solution, $\bar{\Omega}$, the 'Optimal Interpolation formula'. They proved that the function $\bar{\Omega}$ is a spline of degree $k-1$ with exactly $n-k$ knots, and they proved that the corresponding function $\bar{B}$ is the modulus of a perfect spline of degree $k$ with the same $n-k$ knots as $\bar{\Omega}$. The fact that $\bar{B}$ is the modulus of a perfect spline was also discovered by Micchelli, Rivlin and Wino$\operatorname{grad}(1976)$.

The purpose of this paper is to explain how to compute the functions $\bar{\Omega}$ and $\bar{B}$. Our method of computation uses $B$-splines. Therefore, in Section 2 we define $B$ splines. In Section 3 we describe how to compute $\bar{\Omega}$ and in Section 4 we describe our method for computing $\bar{B}$. Finally, in Section 5 we discuss the choice of a suitable value for $k$, and we consider the interesting case when the data points are equally spaced.

The calculations in this paper were performed, in single precision, on the IBM 370/168 computer at Harwell.

Part of this work is from the author's D. Phil. thesis, for Oxford University, which was financed by S. R. C. grant number B/73/794. This paper was written while the author was working at A. E. R. E. Harwell, under an Extramural Research contract with Oxford University. 
2. $B$-Splines. $A B$-spline of degree $k-1, k \geqslant 1$, is a spline function, of the same degree, with $k+1$ knots. It has the important property that it is identically zero outside the range defined by its knots. Consequently, $B$-splines give rise to matrices of band structure which are computationally advantageous.

For the definition of $B$-splines we use the function

$$
g_{k}(x ; y)=(y-x)_{+}^{k-1}= \begin{cases}(y-x)^{k-1}, & x<y, \\ 0, & x \geqslant y,\end{cases}
$$

and denote a sequence of $k+1$ knots by

$$
x_{i}<x_{i+1}<\cdots<x_{i+k-1}<x_{i+k}
$$

The $B$-spline of degree $k-1$, or order $k$, is denoted by $M_{k, i}(x)$ and is defined in the following way. We regard $g_{k}(x ; y)$ as a function of $y$; and, in particular, we consider this function when $y$ takes the values $x_{i}, \ldots, x_{i+k}$. We form the $k$ th divided difference, with respect to $y$, over these values, and using Steffenson's (1927) notation, denote it by $g_{k}\left(x ; x_{i}, \ldots, x_{i+k}\right)$. Then, for $k \geqslant 1, M_{k, i}(x)$ is the function

$$
M_{k, i}(x)=g_{k}\left(x ; x_{i}, \ldots, x_{i+k}\right) .
$$

The $B$-spline $M_{k, i}(x)$ is nonzero only over the interval $x_{i}<x<x_{i+k}$ for the following reasons. By definition $M_{k, i}(x)=0$ when $x \geqslant x_{i+k}$. Moreover, when $x \leqslant x_{i}$, $M_{k, i}(x)$ is the $k$ th divided difference of a polynomial of degree $k-1$ and, therefore, vanishes identically.

For computational purposes it is sometimes useful to use the $B$-splines

$$
N_{k, i}(x)=\left(x_{i+k}-x_{i}\right) M_{k, i}(x),
$$

which are called 'Normalized $B$-splines' (see de Boor (1972) and Cox (1975) for instance). Greville (see the supplement in Schoenberg (1967)) has proved that these $B$ splines have the property that

$$
\sum_{i} N_{k, i}(x)=1
$$

3. To Compute the Optimal Interpolation Formula. In this section we describe how to compute the optimal interpolating function $\bar{\Omega}$ which is the solution to the problem defined in Section 1. We begin by reviewing the properties of $\Omega$ which were proved by Gaffney and Powell (1975) and Gaffney (1976b).

The function $\Omega$ which interpolates values $f_{1}, \ldots, f_{n}$ of a function $f$ at $n$ distinct points $x_{1}<x_{2}<\cdots<x_{n-1}<x_{n}$, and which provides the smallest possible value of $B(x)$ in the error bound

$$
|f(x)-\Omega(x)| \leqslant B(x)\left\|f^{(k)}\right\|_{\infty},
$$

where $\left\|f^{(k)}\right\|_{\infty}$ is bounded, but the value of the bound is unknown, is a spline function, $\bar{\Omega}$, of degree $k-1$. It has exactly $n-k$ knots 


$$
\eta_{1}^{*}<\eta_{2}^{*}<\cdots<\eta_{n-k-1}^{*}<\eta_{n-k}^{*}
$$

which satisfy the inequalities

$$
x_{i}<\eta_{i}^{*}<x_{i+k}, \quad i=1, \ldots, n-k,
$$

and are defined by the conditions

$$
\sum_{j=0}^{n-k}(-1)^{j} \int_{\eta_{j}^{*}}^{\eta_{j+1}^{*}} M_{k, i}(x) d x=0, \quad i=1, \ldots, n-k
$$

where $\eta_{0}^{*}=x_{1}$ and $\eta_{n-k+1}^{*}=x_{n}$. We note that the definition (3.4) implies that the knots $\left\{\eta_{i}^{*}\right\}$ do not depend on the function $f$.

In order to compute $\bar{\Omega}$ we first calculate the $n-k$ knots $\left\{\eta_{i}^{*}\right\}, i=1, \ldots$, $n-k$. This calculation is a special case of that considered by Gaffney (1977a). However, in this case, because the knots $\left\{\eta_{i}^{*}\right\}$ depend only on the data points $\left\{x_{i}\right\}$, there are some simplifications and it is easier to illustrate the type of results that can be expected. Therefore, we include in this section a description of our method for computing the knots $\eta_{1}^{*}, \ldots, \eta_{n-k}^{*}$. It is given in subsection 3.1. When the knots have been calculated the remaining parameters of $\bar{\Omega}$ are linear and we explain how to compute them in subsection 3.2.

3.1. To Compute the Knots of $\bar{\Omega}$. Since conditions (3.4) yield $n-k$ equations for the knots $\left\{\eta_{i}^{*}\right\}, i=1, \ldots, n-k$, we solve the system of equations

$$
\sum_{j=0}^{n-k}(-1)^{j} \int_{\eta_{j}}^{\eta_{j+1}} M_{k, i}(x) d x=0, \quad i=1, \ldots, n-k,
$$

where $\eta_{0}=x_{1}$ and $\eta_{n-k+1}=x_{n}$. Gaffney $(1976 \mathrm{~b})$ has proved that there exists a unique solution to the equations (3.5).

The integral in the middle of Eq. (3.5) may be calculated by using the formula of Gaffney (1976a) together with Cox's (1972) algorithm for evaluating $B$-splines.

When $k=1 \mathrm{Eq}$. (3.5) is linear and in this case it is straightforward to show that the $\operatorname{knots}\left\{\eta_{i}^{*}\right\}$ have the values

$$
\eta_{i}^{*}=\frac{\left(x_{i}+\dot{x}_{i+1}\right)}{2}, \quad i=1, \ldots, n-1
$$

We now consider the solution of Eqs. (3.5) when the value of $k$ is greater than 1. In this case the equations are nonlinear and it is convenient to write them in the vector form

$$
F \eta=0,
$$

where $F$ is the mapping whose $i$ th component $F_{i}(\eta)$ is the left-hand side of Eq. (3.5) and $\eta$ is the vector of unknowns $\eta=\left[\eta_{1}, \ldots, \eta_{n-k}\right]^{T}$. To solve Eq. (3.7) we use Newton's method with some additional precautions. Thus, we require the Jacobian matrix $J(\eta)$ of the system (3.7). It is straightforward to show (see p. 319 in Gaffney (1976b)) that the element in row $p$ and column $q$ of $J(\eta)$ is the quantity 


$$
J_{p, q}(\eta)=2(-1)^{q-1} M_{k, p}\left(\eta_{q}\right)
$$

where $1 \leqslant p, q \leqslant n-k$.

To apply Newton's method we choose an initial approximation $\eta^{(0)}=$ $\left[\eta_{1}^{(0)}, \ldots, \eta_{n-k}^{(0)}\right]^{T}$ to the solution $\eta^{*}$ and then for $t=0,1, \ldots$ we solve the system of linear equations

$$
J\left(\eta^{(t)}\right) \delta^{(t)}=-F \eta^{(t)}
$$

to obtain a new approximation

$$
\eta^{(t+1)}=\eta^{(t)}+\delta^{(t)}
$$

We terminate this iteration either, when the sum of the squares of the residuals

$$
S S\left(\eta^{(t)}\right)=\sum_{i=1}^{n-k}\left[F_{i}\left(\eta^{(t)}\right)\right]^{2}
$$

is less than a prescribed tolerance $\epsilon_{1}$ or when the inequalities

$$
\left(\sum_{i=1}^{n-k}\left[\delta_{i}^{(t)}\right]^{2}\right)^{1 / 2} \leqslant \epsilon\left(\sum_{i=1}^{n-k}\left[\eta_{i}^{(t)}\right]^{2}\right)^{1 / 2}
$$

are satisfied. In practice we choose $\epsilon$ to be the relative machine accuracy, and we let $\epsilon_{1}$ be the value $100(n-k)^{2} \epsilon^{2}$.

In order to solve Eqs. (3.9) the successive matrices $J\left(\eta^{(t)}\right), t=0,1, \ldots$, must be nonsingular. Therefore, (see Gaffney (1976b)) the components of $\eta^{(t)}, t=0,1$, $\ldots$, must be distinct and they must satisfy the inequalities

$$
x_{i}<\eta_{i}^{(t)}<x_{i+k}, \quad i=1, \ldots, n-k ; t \geqslant 0 .
$$

To ensure that $J\left(\eta^{(0)}\right)$ is nonsingular we choose the components of the initial approximation to be the quantities

$$
\eta_{i}^{(0)}=\frac{1}{k+1} \sum_{j=i}^{i+k} x_{j}, \quad i=1, \ldots, n-k,
$$

which are the arithmetic means of the knots of the $B$-splines $M_{k, i}(x), i=1, \ldots, n-$ $k$. In this case when the data points $\left\{x_{i}\right\}, i=1, \ldots, n$, are evenly spaced, and $k=2$, it can easily be verified that the optimal knots $\left\{\eta_{i}^{*}\right\}, i=1, \ldots, n-2$, have the values given by (3.14). Furthermore, when $k \geqslant 3$, we have found that the Newton iterates $\left\{\eta_{i}^{(t)}\right\}, t \geqslant 1$, are in ascending order

$$
\eta_{1}^{(t)}<\eta_{2}^{(t)}<\cdots<\eta_{n-k-1}^{(t)}<\eta_{n-k}^{(t)}, \quad t \geqslant 1 ;
$$

they satisfy conditions (3.13) for all $t \geqslant 1$; and they converge rapidly to the solution $\eta_{i}^{*}, i=1, \ldots, n-k$. However, when the data points are not evenly spaced the above method sometimes provides an approximation which does not satisfy conditions (3.13); and, thus, the iteration breaks down. Therefore, in order to ensure convergence to a solution of Eqs. (3.7) we use a continuation method (see p. 230 in Ortega and Rheinboldt (1970)) together with Newton's method. For example, if the above 
iteration breaks down at the $r$ th step and $J\left(\eta^{(r-1)}\right)$ is nonsingular, then we partition the closed interval $[0,1]$ by a sequence of distinct points

$$
0=\lambda_{r-1}<\lambda_{r}<\cdots<\lambda_{r+p-1}<\lambda_{r+p}=1
$$

and determine suitable values for $\left\{\lambda_{s}\right\}, s=r, \ldots, r+p-1$, so that Newton's method, for solving the equations

$$
T\left(\eta^{(s)}, \lambda_{s}\right) \equiv F \eta^{(s)}-\left(1-\lambda_{s}\right) F \eta^{(s-1)}=0
$$

will converge when the starting approximation is the solution of the equation

$$
T\left(\eta^{(s-1)}, \lambda_{s-1}\right)=0 .
$$

Gaffney (1976b) has proved that this method converges to the solution of Eqs. (3.7) in a finite number of steps. A Fortran subroutine has been written for this computation and it is described, in a companion paper, by Gaffney (1977b).

To solve Eqs. (3.9) we note, from Eq. (3.8) and the definition of the $B$-spline $M_{k, p}(x)$, that the Jacobian matrix $J\left(\eta^{(t)}\right)$ has a band structure. Therefore, our method of solution takes advantage of this fact. Specifically we use Gaussian elimination, without interchanges, to solve (3.9). It is unnecessary to perform any interchanges in the elimination process because of the properties of $\boldsymbol{B}$-splines (see de Boor and Pinkus (1977)). Thus, we take advantage of the sparsity in $J\left(\eta^{(t)}\right)$ by treating it as a band matrix.

To illustrate the type of results which can be expected, we present three examples.

Example 1. For the first example we use the equally spaced data points $x_{i}=i$, $i=1, \ldots, 6$, and let $k=4$. In this case the equations (3.5), for the knots $\eta_{1}^{*}$ and $\eta_{2}^{*}$ are

$$
F_{1}(\eta) \equiv \int_{1.0}^{\eta_{1}} M_{4,1}(x) d x-\int_{\eta_{1}}^{\eta_{2}} M_{4,1}(x) d x+\int_{\eta_{2}}^{6.0} M_{4,1}(x) d x=0, \quad \text { and }
$$

$$
F_{2}(\eta) \equiv \int_{1.0}^{\eta_{1}} M_{4,2}(x) d x-\int_{\eta_{1}}^{\eta_{2}} M_{4,2}(x) d x+\int_{\eta_{2}}^{6.0} M_{4,2}(x) d x=0 .
$$

We used Newton's method to solve Eq. (3.19), and terminated the iteration when the corresponding sum of squares of residuals (3.11) became less than or equal to $4 \cdot 10^{-12}$. Newton's method gives sufficient accuracy after two iterations. The successive iterates are presented in the first two columns of Table 3.1, and the corresponding residuals and sum of squares of residuals are given in the remaining three columns.

Example 2. For the second example we use the data points

$$
x_{i}= \begin{cases}\exp ((i+38)(0.001)), & i=1, \ldots, 11, \\ \exp (0.971+(i-12)(0.001)), & i=12, \ldots, 24,\end{cases}
$$

which are unequally spaced, and we choose $k=6$. In this case the Newton iteration 
(3.9)-(3.10), for solving the corresponding Eqs. (3.7), with the initial approximation

$$
\eta_{i}^{(0)}=\left(\sum_{j=i}^{i+6} x_{j}\right) / 7.0
$$

breaks down. The reason for this is that the correction $\delta^{(0)}$, which is the solution of Eqs. (3.9) with $t=0$, is such that the approximation $\eta^{(0)}+\delta^{(0)}$ does not satisfy the inequalities

$$
x_{i}<\eta_{i}^{(0)}+\delta_{i}^{(0)}<x_{i+k}
$$

for all $i=1, \ldots, n-k$. Thus, we use the continuation method (3.16)-(3.18) for solving (3.7). Specifically, we chose $\lambda_{1}=1 / 2$ and solved the equations (3.16), namely

$$
T\left(\eta^{(1)}, 1 / 2\right) \equiv F \eta^{(1)}-1 / 2 F \eta^{(0)}=0,
$$

using Newton's method with the initial approximation (3.21). After four iterations the corresponding inequality (3.12), with $\epsilon=1.0 \times 10^{-6}$, was satisfied; and we terminated the iteration. We now used this solution as the starting approximation in the iteration (3.9)-(3.10). After a further three iterations we obtained the solution shown in Table 3.2.

TABLE 3.1

\begin{tabular}{|c|c|c|c|c|c|}
\hline$t$ & $\eta_{1}^{(t)}$ & $n_{2}^{(t)}$ & $\left.F_{1} \underline{\underline{n}}^{(t)}\right)$ & $\left.F_{2}{ }^{(t)}\right)$ & $S S\left(\underline{\underline{n}}^{(t)}\right)$ \\
\hline 0 & 3.0000 & 4.0000 & 0.0833 & 0.0833 & $0.138889 .10^{-1}$ \\
1 & 2.9500 & 4.0500 & 0.0013 & 0.0013 & $0.332678 .10^{-5}$ \\
2 & 2.9492 & 4.0508 & 0.0000 & 0.0000 & $0.696332 .10^{-12}$ \\
\hline
\end{tabular}

TABLE 3.2

\begin{tabular}{|r|r|r|r|r|r|r|r|r|l|}
\hline & $i$ & $x_{i}$ & $x_{i+1}$ & $x_{i+2}$ & $x_{i+3}$ & $x_{i+4}$ & $x_{i+5}$ & $x_{i+6}$ & $x_{i+7}$ \\
\hline & 1 & 1.0398 & 1.0408 & 1.0419 & 1.0429 & 1.0439 & 1.0450 & 1.0460 & 1.0471 \\
& 9 & 1.0481 & 1.0492 & 1.0502 & 2.6406 & 2.6432 & 2.6459 & 2.6485 & 2.6512 \\
& 17 & 2.6538 & 2.6565 & 2.6591 & 2.6618 & 2.6645 & 2.6671 & 2.6698 & 2.6725 \\
\hline$t$ & $i$ & $n_{j}^{(t)}$ & $n_{i+1}^{(t)}$ & $n_{j+2}^{(t)}$ & $n_{j+3}^{(t)}$ & $n_{j+4}^{(t)}$ & $n_{j+5}^{(t)}$ & & $s S(\underline{\underline{n}}(t))$ \\
\hline 3 & 1 & 1.0427 & 1.0439 & 1.0450 & 1.0461 & 1.0473 & 1.1270 & & \\
& 7 & 1.3488 & 1.6693 & 2.0251 & 2.3456 & 2.5674 & 2.6481 & & \\
& 13 & 2.6510 & 2.6538 & 2.6565 & 2.6592 & 2.6620 & 2.6650 & & $0.185445 .10^{-7}$ \\
\hline
\end{tabular}

Example 3. This example illustrates the form of the solution, when the data points are evenly spaced and $n$ is very much larger than $k$. We chose $n=100, k=4$ and $x_{i}=i, i=1, \ldots, 100$. Then, with the initial approximation

$$
\eta_{i}^{(0)}=i+2, \quad i=1, \ldots, 96,
$$

Newton's method obtains, after three iterations, the vector $\eta^{*}$ whose components $\eta_{i}^{*}$, 
$i=7, \ldots, 90$, rounded to 4 decimal places, are the values

$$
\eta_{i}^{*}=i+2, \quad i=7, \ldots, 90 .
$$

The remaining components are given, to 4 decimal places, in Table 3.3. The corresponding residuals are less than $2.0 \times 10^{-5}$ and are not shown.

TABLE 3.3

\begin{tabular}{|c|c|c|c|c|c|c|}
\hline$i$ & $\eta_{i}^{*}$ & $n_{i+1}^{*}$ & $n_{i+2}^{*}$ & $n_{i+3}^{*}$ & $n_{i+4}^{*}$ & $n_{i+5}^{*}$ \\
\hline 1 & 2.9332 & 3.9838 & 4.9958 & 5.9989 & 6.9997 & 7.9999 \\
\hline 91 & 93.0001 & 94.0003 & 95.0011 & 96.0042 & 97.0162 & 98.0668 \\
\hline
\end{tabular}

We note that away from the ends of the range the knots are almost at the data points. This behavior is typical when the data points are equally spaced and $n$ is large. In fact, numerical experiment has shown that in this situation the optimal knots, towards the center of the range, have the value

$$
\eta_{i}^{*}=x_{i}+k h / 2
$$

to four decimal places, where $h$ is the distance between data points.

3.2. To Compute the Linear Parameters of $\bar{\Omega}$. When the knots $\eta_{1}^{*}, \ldots, \eta_{n-k}^{*}$ have been calculated, the remaining parameters of $\bar{\Omega}$ are linear, and there are $n$ of them. Their values are determined from the interpolation conditions

$$
\bar{\Omega}\left(x_{i}\right)=f_{i}, \quad i=1, \ldots, n .
$$

In order to compute the linear parameters we write $\bar{\Omega}$ as a linear combination of $n$ normalized $B$-splines, (see Cox (1975), for example). Thus, we introduce an additional $2 k$ knots $\left\{\mu_{i}\right\}, i=1, \ldots, k, n+1, \ldots, n+k$, such that

$$
\mu_{k}=\mu_{k-1}=\cdots=\mu_{1}=x_{1}
$$

and

$$
\mu_{n+k}=\mu_{n+k-1}=\cdots=\mu_{n+1}=x_{n} ;
$$

and we define intermediate values of $\mu_{i}$ by the equation

$$
\mu_{j+k}=\eta_{j}^{*}, \quad j=1, \ldots, n-k .
$$

We now let $N_{k, j}(x)$ denote the normalized $B$-spline with knots $\mu_{j}, \mu_{j+1}, \ldots, \mu_{j+k}$, and write $\bar{\Omega}$ as the sum

$$
\bar{\Omega}(x)=\sum_{j=1}^{n} a_{\dot{j}} N_{k, j}(x) .
$$

The constants $a_{1}, \ldots, a_{n}$ are calculated by solving the linear equations

$$
\sum_{j=1}^{n} a_{j} N_{k, j}\left(x_{i}\right)=f\left(x_{i}\right), \quad i=1, \ldots, n
$$


Schoenberg and Whitney (1953), have proved that because the knots $\eta^{*}$ satisfy conditions (3.3) the coefficient matrix

$$
N=\left[\begin{array}{ccc}
N_{k, 1}\left(x_{1}\right) & N_{k, 2}\left(x_{1}\right) \ldots N_{k, n}\left(x_{1}\right) \\
N_{k, 1}\left(x_{2}\right) & N_{k, 2}\left(x_{2}\right) \ldots N_{k, n}\left(x_{2}\right) \\
\cdot & \cdot & \cdot \\
\cdot & \cdot & \cdot \\
\cdot & \cdot & \cdot \\
N_{k, 1}\left(x_{n}\right) & N_{k, 2}\left(x_{n}\right) \ldots N_{k, n}\left(x_{n}\right)
\end{array}\right]
$$

is nonsingular, and thus Eqs. (3.32) have a unique solution.

In order to solve Eqs. (3.32) we note that the structure of $N$ is similar to the transpose of the Jacobian matrix $J(\eta)$. However, in this case, because the additional knots satisfy conditions (3.28) and (3.29), the first and last rows of $N$ always have only one nonzero, namely $N_{k, 1}\left(x_{1}\right)$ and $N_{k, n}\left(x_{n}\right)$, which have the value 1 . Because $N$ is a band matrix, we use the same algorithm for solving Eqs. (3.32) as we used for solving Eqs. (3.9).

Finally, we note that because the knots of $\bar{\Omega}$ only depend on the data points $x_{1}, \ldots, x_{n}$ the calculation of all the optimal interpolation formulae for several functions that share the same data points $x_{1}, \ldots, x_{n}$ can be greatly reduced, since each approximation has the same knots, namely the components of $\eta^{*}$. Furthermore, if we calculate the linear parameters of one such formula, by for example the above method, and retain the triangular factors of the matrix $N$, we can compute the linear parameters of the remaining formulae by forward and back substitution.

Example. To illustrate our procedure for computing the linear parameters of $\bar{\Omega}$ we apply the above method to the data of Table 3.4 , for the case when $k=4$.

TABLE 3.4

\begin{tabular}{|c|r|r|r|r|r|r|}
\hline$x$ & 1.0 & 2.0 & 3.0 & 4.0 & 5.0 & 6.0 \\
$f(x)$ & -1.0 & 1.0 & 6.0 & 0.0 & 3.0 & -6.0 \\
\hline
\end{tabular}

The optimal knots for the data points $\mathrm{x}=[1.0,2.0,3.0,4.0,5.0,6.0]^{T}$ are given in Table 3.1, and they have the values

$$
\eta_{1}^{*}=2.9492, \quad \eta_{2}^{*}=4.0508 .
$$

To determine the linear parameters of $\bar{\Omega}$, we solve the linear equations

$\left[\begin{array}{llllll}1.0000 & 0.0 & 0.0 & 0.0 & 0.0 & 0.0 \\ 0.1155 & 0.5214 & 0.3295 & 0.0336 & 0.0 & 0.0 \\ 0.0 & 0.1132 & 0.6178 & 0.2690 & 0.0000 & 0.0 \\ 0.0 & 0.0000 & 0.2690 & 0.6178 & 0.1132 & 0.0 \\ 0.0 & 0.0 & 0.0336 & 0.3295 & 0.5214 & 0.1155 \\ 0.0 & 0.0 & 0.0 & 0.0 & 0.0 & 1.0000\end{array}\right]\left[\begin{array}{r}a_{1} \\ a_{2} \\ a_{3} \\ a_{4} \\ a_{5} \\ a_{6}\end{array}\right]=\left[\begin{array}{r}-1.0 \\ 1.0 \\ 6.0 \\ 0.0 \\ 3.0 \\ -6.0\end{array}\right]$

and obtain the values 


$$
\begin{aligned}
& a_{1}=-1.0, \quad a_{2}=-6.5350, \quad a_{3}=14.5919 \text {, } \\
& a_{4}=-8.4579, \quad a_{5}=11.4852, \quad a_{6}=-6.0 \text {. }
\end{aligned}
$$

The result of evaluating the appropriate expression (3.31) for $\bar{\Omega}(x)$, at the points

$$
t_{i}=1.0+0.05(i-1), \quad i=1, \ldots, 101,
$$

and of passing a smooth curve through the values $\bar{\Omega}\left(t_{i}\right), i=1, \ldots, 101$, is shown as the broken curve in Figure 3.1. Also, in this figure we show, from Gaffney (1977a), the range of possible values of the unknown function $f$ when the bound

$$
\left\|f^{(i v)}\right\|_{\infty} \leqslant 100
$$

is valid. The figure shows that in this case, the optimal interpolation formula provides an estimate of the unknown function $f$, which differs only slightly from the best estimate (the mean of the two solid curves in Figure 3.1), that can be obtained using the function values in Table 3.4 and the additional information given by (3.38).

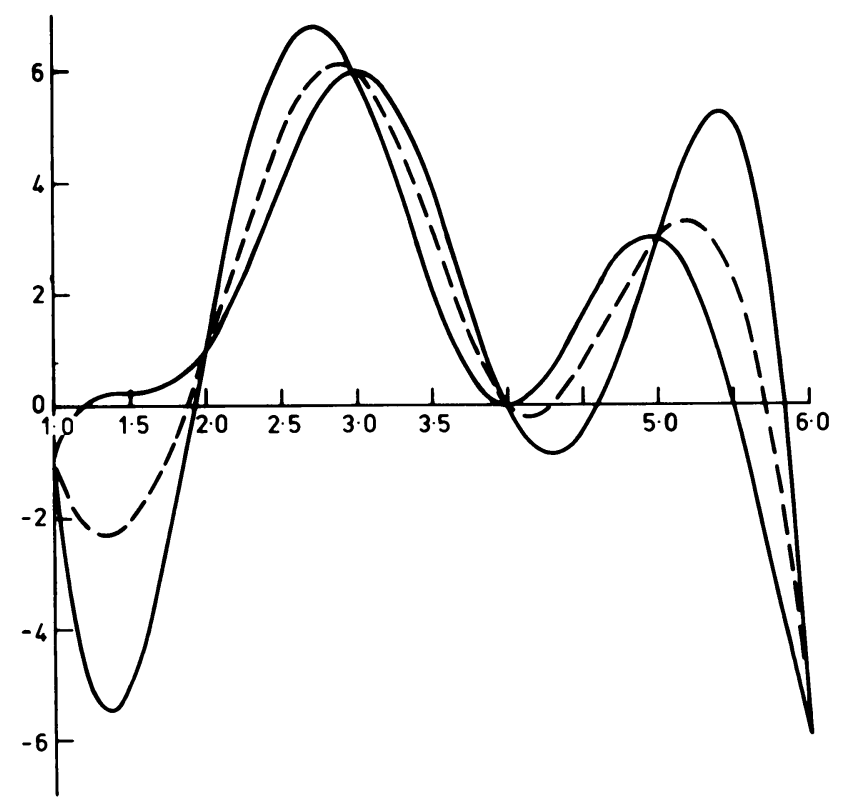

FIGURE 3.1

The broken curve is the optimal interpolation formula $\bar{\Omega}(x)$ for the data of Table 3.4. The solid curves define the range of possible values of $f$ when the bound $\left\|f^{\mathrm{iv}}\right\|_{\infty} \leqslant 100$ is valid

4. To Compute the Function $\bar{B}$. In this section we describe how to compute the function $\bar{B}$ which is associated with the optimal interpolating function $\bar{\Omega}$.

We first state the properties of $\bar{B}$ which were proved by Gaffney and Powell (1975) and Gaffney (1976b).

The function $\bar{B}$ which provides the smallest possible value of $B(x), x_{1} \leqslant x \leqslant x_{n}$, in the error bound (1.1) is the modulus of a perfect spline $\bar{\beta}$, of degree $k$, which has $n-k$ knots $\eta_{1}^{*}, \ldots, \eta_{n-k}^{*}$ and satisfies the conditions 


$$
\bar{\beta}\left(x_{i}\right)=0, \quad i=1, \ldots, n,
$$

and

$$
\vec{\beta}^{(k)}(x)= \begin{cases}1, & x_{1} \leqslant x<\eta_{1}^{*}, \\ (-1)^{i}, & \eta_{i}^{*} \leqslant x<\eta_{i+1}^{*}, \quad i=1, \ldots, n-k-1 . \\ (-1)^{n-k}, & \eta_{n-k}^{*} \leqslant x \leqslant x_{n} .\end{cases}
$$

To compute $\bar{B}$ we first calculate the knots $\eta_{1}^{*}, \ldots, \eta_{n-k}^{*}$ as described in Section 3. We then determine the remaining $\underline{n+1}$ linear parameters of $\bar{\beta}$ so that conditions (4.1) and (4.2) are satisfied. In order to do this we note that any spline function, $S$, of degree $k$, which has the $n-k$ knots $\eta_{1}^{*}, \ldots, \eta_{n-k}^{*}$, and which has the properties

$$
S(x) \not \equiv 0, \quad x_{1} \leqslant x \leqslant x_{n},
$$

and

$$
S\left(x_{i}\right)=0, \quad i=1, \ldots, n,
$$

is a constant nonzero multiple of $\bar{\beta}$. Therefore, if $x^{*}$ is any fixed value of $x$ in any one of the intervals $\eta_{i}^{*}<x<\eta_{i+1}^{*}, 0 \leqslant i \leqslant n-k$, where $\eta_{0}^{*}=x_{1}$ and $\eta_{n-k+1}^{*}=x_{n}$, then the derivative

$$
S^{(k)}\left(x^{*}\right)=\left.S^{(k)}(x)\right|_{x=x^{*}}
$$

is nonzero and the equation

$$
\bar{\beta}(x)=(-1)^{i}\left[S^{(k)}\left(x^{*}\right)\right]^{-1} S(x)
$$

is valid for all $x$ in $\left[x_{1}, x_{n}\right]$.

Thus, to compute $\bar{B}$ we first obtain a spline $S$ of degree $k$, which has the knots $\eta_{1}^{*}, \ldots, \eta_{n-k}^{*}$, and which satisfies the conditions (4.3) and (4.4). Then, we let $x^{*}$ have the value

$$
x^{*}=1 / 2\left(\eta_{r}^{*}+\eta_{r+1}^{*}\right)
$$

where the integer $r$ is for convenience defined by the expression

$$
\eta_{r+1}^{*}-\eta_{r}^{*}=\operatorname{Min}_{0 \leqslant i \leqslant n-k}\left(\eta_{i+1}^{*}-\eta_{i}^{*}\right),
$$

and we calculate $S^{(k)}\left(x^{*}\right)$ by using the formula due to de Boor (1972). Finally, we compute $\bar{B}(x)$ from the formula

$$
\bar{B}(x)=\left|\left[S^{(k)}\left(x^{*}\right)\right]^{-1} S(x)\right| .
$$

We have applied the above method to compute the function $\bar{B}$ for the data of Table 3.4 in Section 3. It is shown in Figure 4.1.

5. Discussion. In this section we discuss the choice of a suitable value for $k$; and we consider the interesting case when the data points $\left\{x_{i}\right\}, i=1, \ldots, n$, are equally spaced. 


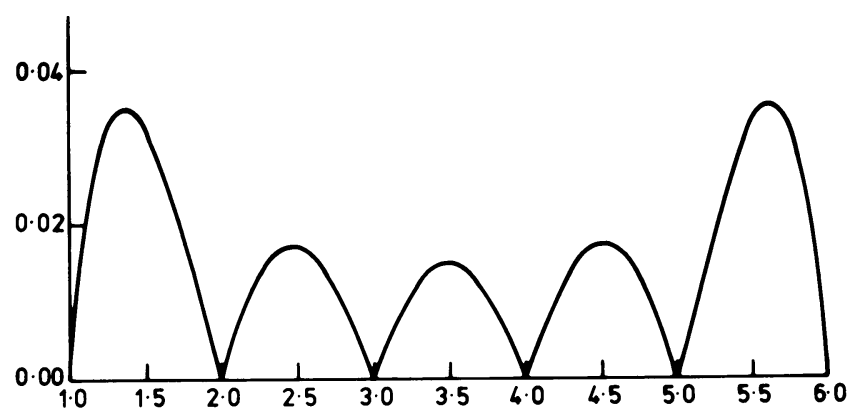

FIGURE 4.1

The function $\bar{B}$ for the data of Table 3.4

When $k=n$, the optimal interpolation formula $\bar{\Omega}(x)$ is simply the Lagrange interpolating polynomial of degree $n-1$ which passes through the given function values $f_{1}, \ldots, f_{n}$. If the approximation is required to be accurate throughout the interval $\left[x_{1}, x_{n}\right]$, then in general a single interpolating polynomial is unsuitable. For example, if the data points are evenly spaced and the value of $n$ is large, then the approximation to $f$ near the ends of the range is often very poor, while towards the center of the range it is usually better. This is especially true if the function $f$ does not behave like a polynomial. In general, we recommend that the value of $k$ should be chosen very much smaller than the number of data points $n$. In this way the spline $\bar{\Omega}$ is composed of a number of piecewise polynomials and, therefore, nonpolynomial like behavior, exhibited by the data, can be accounted for. When the value of $k$ is 1 the optimal knots are the quantities given by (3.6). Therefore, $\bar{\Omega}(x)$ is just the piecewise constant function

$$
\bar{\Omega}(x)= \begin{cases}f_{1}, & x_{1} \leqslant x \leqslant \eta^{*}, \\ f_{i+1}, & \eta_{i}^{*} \leqslant x \leqslant \eta_{i+1}^{*}, \quad i=1, \ldots, n-2, \\ f_{n}, & \eta_{n-1}^{*} \leqslant x \leqslant x_{n},\end{cases}
$$

which, in general, is not accurate enough to provide a satisfactory approximation to $f$. When $k=2$ and the data points are evenly spaced, the optimal knots are given by (3.14) and they have the values

$$
\eta_{i}^{*}=x_{i+1}, \quad i=1, \ldots, n-2 .
$$

Thus, it follows that the optimal interpolation formula $\bar{\Omega}(x)$, is the piecewise linear function

$$
\begin{aligned}
\bar{\Omega}(x)=\left(\frac{x_{i+1}-x}{x_{i+1}-x_{i}}\right) f_{i}+\left(\frac{x-x_{i}}{x_{i+1}-x_{i}}\right) & f_{i+1}, \\
x_{i} & \leqslant x \leqslant x_{i+1}, i=1, \ldots, n-1 .
\end{aligned}
$$

Usually, piecewise linear functions are not suitable for approximating smooth functions to high accuracy. In practice we have found that, for most functions, the choice $k=3$ or 4 is sufficient to provide an approximation $\bar{\Omega}$ which resembles one that might be estimated by an experienced computer user. The optimal interpolating 
spline $\bar{\Omega}$ has the further nice property that if the data is taken from a polynomial of degree less than $k$, then $\bar{\Omega}$ reproduces this polynomial exactly.

We now consider the interesting case, which occurs often in practice, of equally spaced data points. At the end of Example 3 in Section 3 we noted that when the data points $\left\{x_{i}\right\}, i=1, \ldots, n$, are equally spaced and $n$ is large; the optimal knots $\left\{\eta_{i}^{*}\right\}$, towards the center of the range, have the value

$$
\eta_{i}^{*} \simeq x_{i}+k h / 2,
$$

where $h$ is the distance between the data points. We also noted, in Eq. (5.2), that when $k=2$ the knots are at the data points $\left\{x_{i+1}\right\}, i=1, \ldots, n-2$. In general, when $k$ is even and greater than 2 , some of the knots are almost at the data points. In order to test the difference between $\bar{\Omega}$ and a spline $S$ which interpolates the function values $f_{i}, i=1, \ldots, n$, and whose $n-k$ knots $\left\{\eta_{i}\right\}$ are at the interior data points, i.e.

$$
\eta_{i}=x_{i}+k h / 2, \quad i=1, \ldots, n-k,
$$

we approximated the function

$$
f(x)=-x e \log _{e} x
$$

over the interval $0.0 \leqslant x \leqslant 1.0$. The graph of $f(x)$ is shown in Figure 5.1.

We chose $k=4,6$ and 8 with 22 equally spaced, data points. The optimal knots $\left\{\eta_{i}^{*}\right\}$ and the knots $\left\{\eta_{i}\right\}$ are given in ascending order, correct to four decimal places, in Table 5.1.

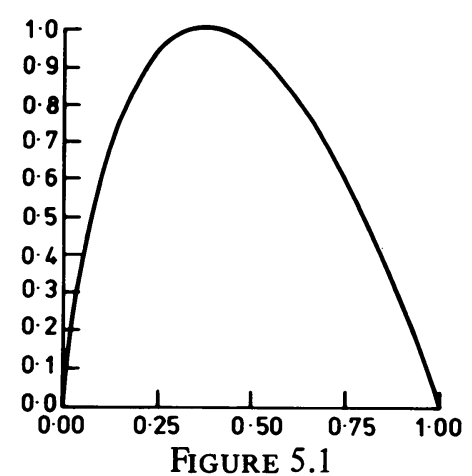

$$
f(x)=-x e \log _{e} x
$$

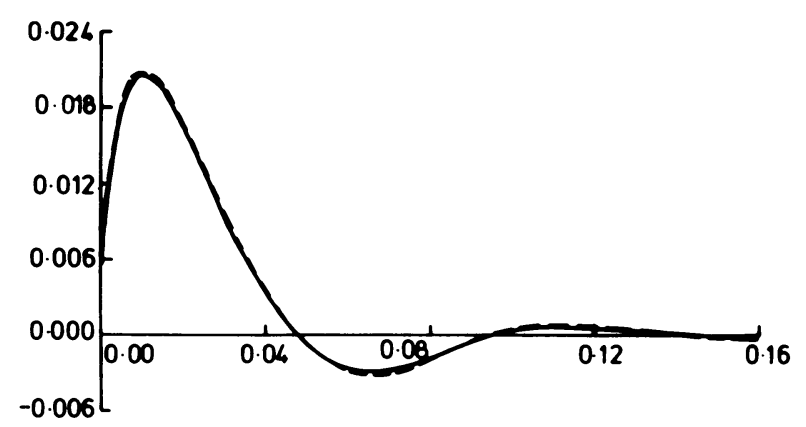

FIGURE 5.2. $-d_{4}$ and $e_{4}---$ 


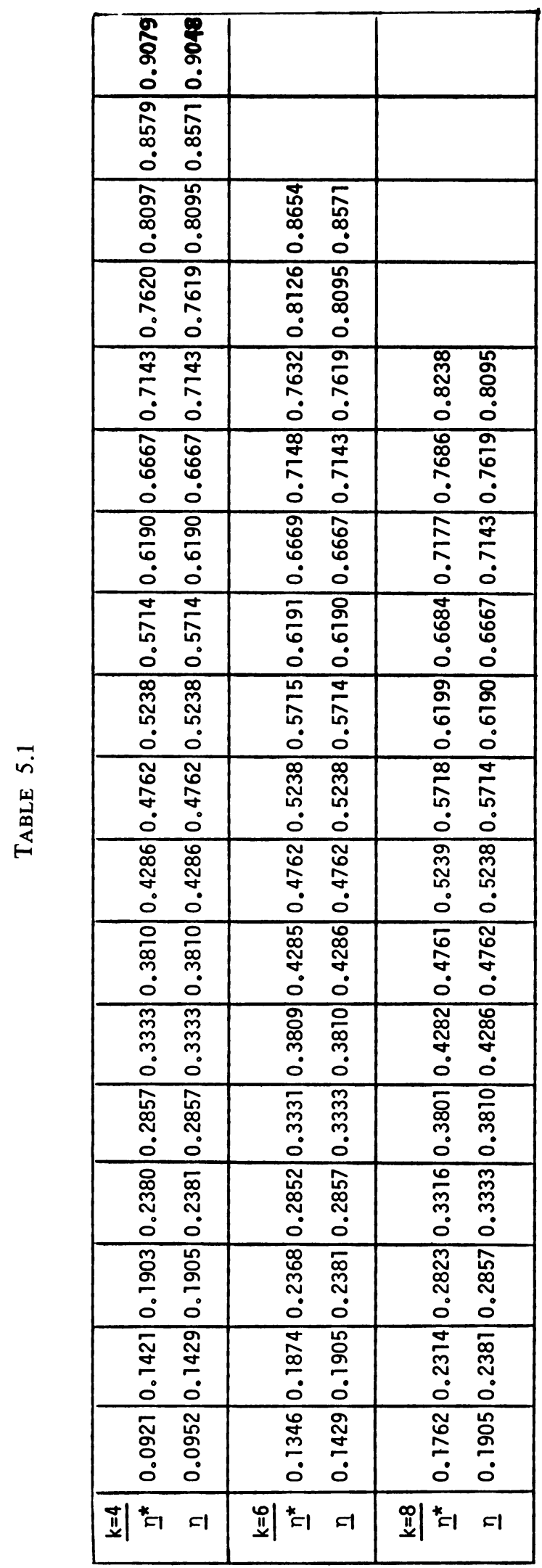




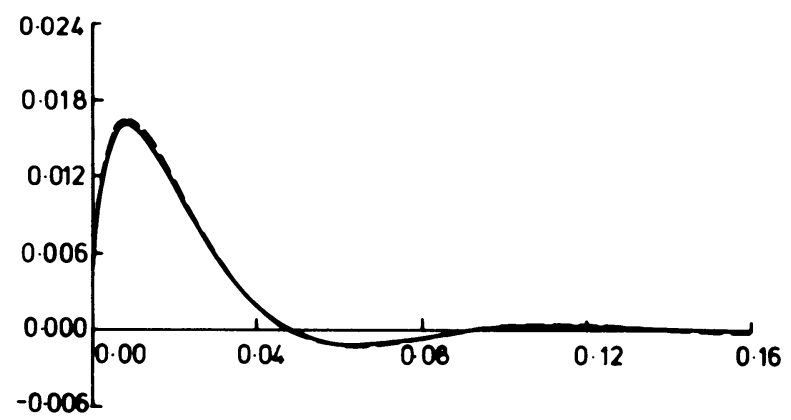

FIGURE 5.3. $-d_{6}$ and $e_{6}---$

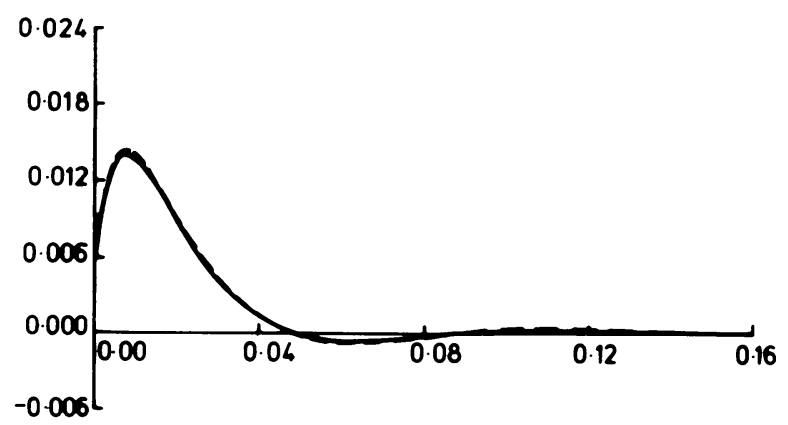

FIGURE 5.4. $-d_{8}$ and $e_{8}---$

To measure the difference between the approximations of $f$ we calculated the error functions

$$
e_{k}=f-\bar{\Omega}
$$

and

$$
d_{k}=f-S
$$

for some values of $x$. These results are given in Figures 5.2-5.4. When $x$ is in the interval $0.16<x \leqslant 1.0$, the functions $e_{k}$ and $d_{k}$ are zero to three decimal places for $k=4,6$ and 8 .

Now, because the first derivative of $f$ has a logarithmic singularity at $x=0$, we expect the error in the approximation of $f$ near the origin to be relatively large. Figures 5.2-5.4 show that this is the case, but more importantly they show that the approximations $\bar{\Omega}$ and $S$ do not differ much. This fact is important because it is easier to calculate the spline $S$. Moreover, although we have tested few problems, we believe that if an approximation to a function $f(x), x_{1} \leqslant x \leqslant x_{n}$, is required to be accurate in an interval away from the ends of the range then, provided the number of equally spaced data points in $\left[x_{1}, x_{n}\right]$ is sufficiently large, there is very little to be gained in preferring the optimal cubic spline approximation $\bar{\Omega}$, to the cubic spline $S$ which interpolates the data and has its knots given by expression (5.5).

In this paper we have explained how to compute the optimal interpolation formula $\bar{\Omega}(x)$. We have shown that the $n-k$ knots of $\bar{\Omega}(x)$ can be calculated easily by solving a system of nonlinear equations. Moreover, the $n$ linear parameters of 
$\bar{\Omega}(x)$ are obtained, from the interpolation conditions, by solving a system of linear equations whose coefficient matrix has a band structure. Therefore, the optimal interpolation formula is ideally suited for computer calculation. Our results indicate that $\bar{\Omega}(x)$ is a very good solution to the interpolation problem, where we measure goodness by the appearance of the fitted curve. Furthermore, although the interpolation problem requires the $k$ th derivative of $f(x)$ to be bounded, the optimal interpolation method has worked entirely satisfactorily for functions which do not satisfy this condition. For instance there may be singularities at the ends of the range.

A Fortran subroutine for computing the optimal interpolation formula has been written by Gaffney (1977b).

Acknowledgements. It is a pleasure to thank my supervisor Professor M. J. D. Powell, for his help and guidance throughout the development of my thesis, and for encouraging me to write this paper. I also wish to thank Dr. J. K. Reid for reading an earlier draft of this paper and making suggestions which have improved its presentation.

Computer Science and Systems Division

Building 8.9, A.E.R.E. Harwell

Oxfordshire, England

M. G. COX,(1972), "The numerical evaluation of B-splines," J. Inst. Math. Appl., v. 10, pp. 134-149.

M. G. COX (1975), “An algorithm for spline interpolation,” J. Inst. Math. Appl., v. 15, pp. 95-108.

C. de BOOR (1972), "On calculating with B-splines," J. Approximation Theory, v. 6, pp. 50-62.

C. de BOOR \& A. PINKUS (1977), "Backward error analysis for totally positive linear systems," Numer. Math., v. 27, pp. 485-490.

P. W. GAFFNEY (1976a), "The calculation of indefinite integrals of $B$-splines," J. Inst.

Math. Appl., v. 17, pp. 37-41.

P. W. GAFFNEY (1976b), Optimgl Interpolation, D. Phil. Thesis, Oxford University.

P. W. GAFFNEY (1977a), The Range of Possible Values of $f(x)$, A.E.R.E. Report C.S.S. 51.

P. W. GAFFNEY (1977b), Fortran Subroutines for Computing the Optimal Interpolation Formula, A.E.R.E. Report No. R.8781.

P. W. GAFFNEY \& M. J. D. POWELL (1975), “Optimal interpolation," Numerical Analysis (Proc. Conf., Univ. of Dundee, 1975), G. A. Watson (Editor), Lecture Notes in Math., vol. 506, Springer-Verlag, Berlin and New York.

C. A. MICCHELLI, T. J. RIVLIN \& S. WINOGRAD (1976), "The optimal recovery of smooth functions," Numer. Math., v. 26, pp. 191-200.

J. M. ORTEGA \& W. C. RHEINBOLDT (1970), Iterative Solution of Nonlinear Equations in Several Variables, Academic Press, New York.

I. J. SCHOEN BERG (1967), "On spline functions," Inequalities (Proc. Sympos., WrightPatterson Air Force Base, Ohio, 1965), Oved Shisha (Editor), Academic Press, New York, pp. 255-291.

I. J. SCHOENBERG \& A. WHITNEY (1953), “On Pólya frequency functions. III, The positivity of translation determinants with an application to the interpolation problem by spline curves," Trans. Amer. Math. Soc., v. 74, pp. 246-259.

J. F. STEFFENSON (1927), Interpolation, Chelsea, New Yn-- 